\title{
Estudo transversal sobre trabalho e comportamentos de risco à saúde entre escolares de zona rural do Rio Grande do Sul, 2010*
}

doi:10.5123/S1679-49742020000300006

\author{
Cross-sectional study on work and health risk behaviors among school students in a rural \\ area of Rio Grande do Sul State, Brazil, 2010
}

\section{Estudio transversal sobre trabajo y conductas de riesgo a la salud entre niños estudiantes en zona rural de Rio Grande do Sul, Brasil, 2010}

\author{
Chirle de Oliveira Raphaelli' - (1) orcid.org/0000-0001-6398-3567 \\ Mario Renato de Azevedo Júnior ${ }^{2}$ - (D) orcid.org/0000-0001-9731-6447 \\ Helen Gonçalves ${ }^{3}$ - (D) orcid.org/0000-0001-6470-3352 \\ Pedro Curi Hallal² - (D orcid.org/0000-0003-1470-6461 \\ ${ }^{1}$ Universidade Federal de Pelotas, Faculdade de Agronomia Eliseu Maciel, Pelotas, RS, Brasil \\ ${ }^{2}$ Universidade Federal de Pelotas, Escola Superior de Educação Física, Pelotas, RS, Brasil \\ ${ }^{3}$ Universidade Federal de Pelotas, Faculdade de Medicina, Pelotas, RS, Brasil
}

\section{Resumo}

Objetivo: avaliar a associação entre trabalho e comportamentos de risco à saúde entre escolares de zona rural. Métodos: estudo transversal com escolares do $5^{\circ}$ ao $8^{\circ}$ ano do ensino fundamental das escolas municipais de Barão do Triunfo, RS, Brasil, em 2010; utilizou-se questionário autoaplicável; caracterizou-se o perfil de trabalho como 'trabalhadores atuais', 'trabalhadores prévios' e 'não trabalhadores'; revelaram-se comportamentos de risco à saúde o tabagismo, o consumo de álcool, o excesso de peso e a inatividade física ( $\leq 300$ minutos semanais). Resultados: dos 339 participantes menores de 16 anos (89,9\%), 82,3\% eram trabalhadores atuais; entre os trabalhadores, predominaram serviços domésticos (39,8\%) e agricultura (29,9\%); 24,8\% estavam com excesso de peso, 51,2\% inativos no lazer, 6,6\% fumavam; e 27,0\% haviam consumido álcool nos últimos 30 dias, principalmente trabalhadores, comparados a não trabalhadores $(\mathrm{p}=0,02)$. Conclusão: oito em cada dez escolares trabalhava, e a maioria destes estava em trabalho infanto-juvenil; consumo de álcool foi o comportamento de risco à saúde mais frequente.

Palavras-chave: Comportamento do Adolescente; Saúde; Trabalho Infantil; Bebidas Alcoólicas; Estudos Transversais.

*Artigo derivado da dissertação de mestrado intitulada 'Associação entre comportamentos de risco à saúde de pais e irmãos e de adolescentes escolares de zona rural', defendida por Chirle de Oliveira Raphaelli junto ao Programa de Pós-Graduação em Educação Física da Escola Superior de Educação Física, Universidade Federal de Pelotas, em 2011.

Endereço para correspondência:

Chirle de Oliveira Raphaelli - Universidade Federal de Pelotas, Faculdade de Agronomia Eliseu Maciel, Programa de PósGraduação em Ciência e Tecnologia de Alimentos, Campus Capão do Leão, s/n, Caixa Postal 354, Pelotas, RS, Brasil. CEP: $96010-900$ E-mail: chirleraphaelli@hotmail.com 


\section{Introdução}

Atualmente, 152 milhões de crianças e adolescentes (menores de 18 anos) no mundo são economicamente ativos, incluindo aqueles em situação de trabalho ilegal e no setor informal da economia. ${ }^{1,2}$ Cerca de $70 \%$ das crianças e adolescentes no mundo, envolvidos no trabalho, estão no setor agrícola, $17 \%$ no serviços e $12 \%$ na indústria, e um terço se ocupa com atividades familiares não remuneradas. ${ }^{1}$ Pobreza, desemprego e crença na contribuição do trabalho para o crescimento pessoal eis alguns dos fatores que levam as famílias, especialmente da zona rural, ${ }^{3}$ a utilizar a mão de obra dos filhos como forma de geração de renda. ${ }^{4}$ Torna-se difícil distinguir entre o que é participação esporádica em atividades de trabalho e trabalho infantil propriamente. ${ }^{5}$ Estes aspectos, entre outros, fazem do trabalho de menores na zona rural, especialmente na agricultura familiar e no âmbito doméstico, um permanente desafio, seja no âmbito legal, seja no da saúde. ${ }^{3}$

\section{Pobreza, desemprego e crença na contribuição do trabalho para o crescimento pessoal eis alguns dos fatores que levam as famílias, especialmente da zona rural, a utilizar a mão de obra dos filhos como forma de geração de renda.}

0 Brasil é signatário de acordos internacionais pela erradicação do trabalho infantil ou infanto-juvenil e cumprimento dos direitos dos adolescentes que desenvolvam alguma atividade laboral. ${ }^{6} 0$ trabalho infantil é aquele realizado por indivíduos menores de 16 anos de idade, independentemente da sua categoria ocupacional, exceto na condição de aprendiz - quando a idade mínima passa a ser de 14 anos. 0 país conta com o Estatuto da Criança e do Adolescente para garantir esses direitos, e com ações governamentais para erradicar o trabalho infantil, minimizar os agravos à saúde e inserir esses indivíduos em atividades escolares e sociais.?

As atividades laborais infantis, de elevada prevalência no país, ${ }^{8}$ têm-se associado a consequências importantes para a saúde, ${ }^{5}$ a destacar a exposição constante a riscos de acidentes, desenvolvimento de doenças ocupacionais, estresse, exaustã $0^{9,10}$ e interferências na vida escolar, como evasão e repetência. ${ }^{11}$ Entre os adolescentes e estudantes que trabalham, 0 consumo de tabaco e 0 uso de álcool ${ }^{12}$ são também comportamentos preocupantes, frequentemente encontrados em residentes da zona rural. ${ }^{13}$

Apesar da relevância desses comportamentos de risco à saúde, durante a infância e adolescência e no decorrer da vida, poucos trabalhos são desenvolvidos com populações rurais tendo por objetivo investigar a relação entre atividade laboral, trabalho infantil e comportamentos de risco. Quando o adolescente participa de uma atividade laboral em condições de segurança, alguns aspectos positivos têm-se destacado como contribuição para o desenvolvimento psicológico, aumento da autoestima e aprendizado de habilidades e experiências individuais importantes para a socialização. Além do mais, autores descrevem que adolescentes trabalhadores de zona urbana se encontram mais fisicamente ativos ${ }^{14} \mathrm{e}$ com peso adequado para a idade, quando comparados aos não trabalhadores. ${ }^{15}$

Em localidades rurais, essas atividades podem ser confundidas com as laborais, e os estudos não avaliaram as atividades realizadas no tempo livre dos adolescentes, importantes para prevenir problemas de saúde., ${ }^{910} \mathrm{Com}$ base no exposto, existe a necessidade de se investigar a relação entre o trabalhar e os comportamentos de risco à saúde, haja vista a inexistência de estudos com esses desfechos agrupados.

0 objetivo do presente estudo foi avaliar a associação entre trabalho e comportamentos de risco à saúde entre escolares de uma zona rural.

\section{Métodos}

Estudo transversal de base escolar realizado em 2010, no município de Barão do Triunfo, localizado na região carbonífera do estado do Rio Grande do Sul, Brasil.

Com sua economia baseada na fumicultura, seguida pela bovinocultura, o município gaúcho apresenta um dos menores índices de desenvolvimento humano $(\mathrm{IDH}=0,61)$ do estado ${ }^{16} \mathrm{Em} 2010$, Barão do Triunfo contava com 7.018 habitantes, dos quais $90 \%$ residentes na zona rural, oito escolas municipais localizadas na zona rural e duas na zona urbana. ${ }^{16}$ Entre sua população de adolescentes, $52,9 \%$ eram do sexo masculino, 50,1\% tinham 10 a 14 anos e $3,4 \%$ se encontravam fora da escola.

0 estudo partiu da consideração de todos os escolares matriculados do $5^{\circ}$ ao $8^{\circ}$ ano do ensino fundamental, entre 10 e 19 anos de idade, distribuídos em três escolas da rede municipal de educação, que possuíam ensino fundamental completo, localizadas na zona rural. De acordo com o Censo Escolar de 2010, ${ }^{16} 412$ escolares dessa 
faixa etária estavam cadastrados no sistema de ensino municipal, e todos foram incluídos na pesquisa.

Após autorização da Prefeitura local, foram contatados os gestores das escolas e os pais dos alunos. Obtida autorização de ambos, a coleta dos dados aconteceu entre março e setembro de 2010. Os dados de identificação do escolar (nome, idade, série e contato), coletados a partir de consulta à relação de alunos das séries escolares, foram disponibilizados pela Secretaria Municipal de Educação.

Foi utilizado instrumento autoaplicável, previamente testado em amostra similar e não inclusa neste estudo. Utilizado para coleta de dados em um projeto acadêmico de mestrado, o instrumento em questão continha blocos com informações sociodemográficas e sobre trabalho, saúde, alimentação, atividade física, uso de fumo e de álcool. A coleta de dados aconteceu em duas etapas: (i) a aplicação do questionário; e (ii) a aferição de medidas. Inicialmente, foram efetuadas pelo menos três visitas a cada sala de aula, para explicação dos objetivos da pesquisa e aplicação do questionário pelo pesquisador responsável. Nessas ocasiões, foram explanados os objetivos do estudo e dadas orientações sobre como responder ao instrumento. Foi garantido aos participantes o sigilo das informações prestadas por eles; 0 tempo médio para a aplicação do questionário foi de 40 minutos. Os desfechos de interesse foram os comportamentos de risco à saúde (consumo de tabaco, consumo de bebida alcoólica, excesso de peso e inatividade física no lazer), e a variável de exposição principal, qual seja, o perfil de trabalho.

Para os consumos de tabaco e de álcool, foram utilizadas questões adotadas no instrumento do Centro Brasileiro de Informações sobre Drogas Psicotrópicas: perguntou-se ao ou à escolar se, no decorrer do último mês, havia consumido cigarros (de filtro, de palha etc., ainda que uma ou duas tragadas) e se havia consumido bebida alcoólica (por exemplo, cachaça, cerveja, uísque, vodca, vinho etc., mesmo que um ou dois goles). Considerou-se a resposta positiva, para ambos os comportamentos, quando o escolar havia consumido a substância nos últimos 30 dias, independentemente do número de cigarros e do número de doses de bebida alcoólica consumidos. ${ }^{17}$

Em um segundo momento, foram aferidas as medidas de peso e altura, para avaliar o excesso de peso: sobrepeso e obesidade, enquanto comportamento de risco à saúde. Para o peso, foi utilizada balança digital da marca Plenna (até $150 \mathrm{~kg}$ - precisão de 100g); e para a altura em pé, estadiômetro de modelo portátil, da marca Sanny (até
$210 \mathrm{~cm}$ - precisão de $1 \mathrm{~mm})$. As medidas foram tomadas nas escolas, em local reservado, com os escolares vestidos de roupas leves, descalços e em posição ortostática. A tomada dessas medidas foi efetuada duas vezes, encarregada a docentes (previamente treinados durante 8 horas); e foram repetidas quando apresentavam mais de $10 \%$ de diferença entre as duas aferições no mesmo escolar. 0 controle de qualidade foi realizado em $10 \%$ das medidas. 0 excesso de peso foi obtido pelo índice de massa corporal (IMC), classificado com base no percentil $\geq 85 \%$ como parâmetro, segundo idade e sexo. ${ }^{18}$

0 nível de atividade física no lazer foi avaliado investigando-se as atividades realizadas nos sete dias anteriores à aplicação do questionário, excluídas a atividade física escolar e a realizada nos domínios de ocupação, doméstica e de deslocamento. Questões validadas por Bastos et al. (2008) ${ }^{19}$ captaram a frequência e duração de cada atividade física realizada (por exemplo: nadar, jogar bola/futebol, basquetebol etc.). Para classificar os escolares como fisicamente inativos, utilizou-se o ponto de corte de 300 minutos ou menos de atividade por semana.

0 perfil de trabalho, variável de exposição principal, foi definido como qualquer atividade laboral regular, remunerada ou não, domiciliar ou não, referida pelo adolescente. ${ }^{2}$ Essa definição inclui tanto o auxílio em resposta às demandas domésticas (cuidado da casa dos pais ou de outras famílias; cuidado de irmãos menores ou outras crianças) como 0 trabalho formal remunerado e as atividades informais - por exemplo, as atividades desenvolvidas na agricultura e/ou pecuária, em casa ou fora de casa. Foi considerado trabalho infantil quando 0 adolescente menor que 16 anos referiu trabalhar ou realizar qualquer atividade laboral nos últimos 30 dias, incluindo tarefas domésticas, cuidado de crianças, cuidado de animais e trabalho na agricultura. ${ }^{7}$ Foram considerados trabalhadores prévios aqueles que trabalharam alguma vez na vida, porém não estavam trabalhando nos últimos 30 dias; como não trabalhadores, aqueles que nunca trabalharam na vida; e como trabalhadores atuais, os que trabalharam nos últimos 30 dias. Para análise de dados, foram utilizadas duas categorias de trabalho atual (sim; não).

Por meio do questionário apresentado aos escolares, foram investigadas suas características sociais e demográficas e sua autoavaliação de saúde, suas características do trabalho e comportamentos de risco para a própria saúde. Primeiramente, são descritas aqui as características sociodemográficas e a forma de autoavaliação de saúde investigadas: 
a) idade (em anos: 10 a 13; 14 a 15; 16 ou mais);

b) sexo (masculino; feminino);

c) raça/cor da pele (branca; não branca); $\mathrm{e}$

d) autoavaliação de saúde (obtida a partir da questão Como você considera a sua saúde? e as seguintes opções de resposta: excelente; muito boa; boa; regular ou ruim).

Quanto às características do trabalho - dos escolares trabalhadores atuais -, perguntou-se:

a) idade quando começou a trabalhar (em anos: até $11 ; 11$ a $12 ; 13$ ou mais);

b) local da atividade laboral (casa; fora de casa; ambos);

c) tipo de atividade laboral (serviços domésticos/cuidado de irmãos; serviços de agricultura/pecuária; outras atividades);

d) número de horas trabalhadas por dia (até 4; 5 a $8 ; 9$ ou mais);

e) número de dias de trabalho por semana (1 a 5 ; 6 a 7$) ; \mathrm{e}$

f) remuneração em dinheiro (sim; não).

Os comportamentos de risco à saúde avaliados foram:

a) consumo de tabaco no último mês (sim; não);

b) consumo de álcool no último mês (sim; não);

c) excesso de peso (sim; não); $\mathrm{e}$

d) inatividade física semanal no lazer (sim; não).

Como, por questões logísticas, a pesquisa foi realizada na escola. Anteriormente, foi enviado a todos os responsáveis um questionário para autopreenchimento, de fácil compreensão, contendo perguntas sobre renda mensal familiar (em salários mínimos) e escolaridade dos pais (em anos completos de estudo). Junto ao instrumento enviado aos responsáveis, estava anexado o Termo de Consentimento Livre e Esclarecido.

Foi realizada a dupla digitação dos dados no software Epi Info 6.0 para correção de erros pelo Validate. Foram calculadas as prevalências e respectivos intervalos de confiança do trabalho e dos comportamentos de risco à saúde, de acordo com variáveis de exposição, com uso do software Stata 11.0 com nível de 0,05 de significância. Posteriormente, foram verificadas as diferenças de prevalências de trabalho (nunca; prévio; atual) entre os subgrupos dos comportamentos de risco, pelo teste do qui-quadrado. Análises adicionais foram conduzidas considerando-se duas categorias de trabalho: trabalho atual (sim; não), pela regressão de Poisson, com estimativa da variância robusta para avaliar associação entre os comportamentos de risco à saúde; e com trabalho atual, ajustado para as variáveis 'sexo' e 'idade'.
Os adolescentes participaram da pesquisa após seu consentimento verbal e a autorização dos pais/responsáveis mediante assinatura do Termo de Consentimento Livre e Esclarecido. 0 estudo foi autorizado pelo gestor local e aprovado pelo Comitê de Ética em Pesquisa com seres humanos da Escola Superior de Educação Física da Universidade Federal de Pelotas (CEP/ESEF/UFPel): Protocolo $\mathrm{n}^{0} 068$, de 27 de novembro de 2009. Após a autorização do CEP/ESEF/UFPel, foi realizada uma reunião com as diretoras das três escolas municipais, quando cada uma assinou um termo autorizando o início da coleta de dados em sua respectiva instituição de ensino.

\section{Resultados}

Dos 412 escolares elegíveis para o estudo, 377 foram entrevistados e 375 tiveram suas medidas antropométricas coletadas, perfazendo um total de $9 \%$ de perdas (adolescentes ausentes da sala de aula no momento da coleta) e recusas. Do total de participantes, $60,8 \%$ tinham entre 10 e 13 anos de idade, 53,0\% eram do sexo masculino e $54,4 \%$ de raça/cor da pele branca (dados não apresentados em tabelas). 0 percentual de excesso de peso foi de $24,8 \%, 51,2 \%$ eram inativos no lazer, 6,6\% fumaram e 27,0\% consumiram álcool nos 30 dias que antecederam a entrevista (dados não apresentados em tabelas).

Em relação ao trabalho, $82,1 \%\left(\mathrm{IC}_{95 \%} 77,0 ; 86,0\right)$ eram trabalhadores atuais, 9,6\% (IC $\left.{ }_{95 \%} 7,0 ; 13,0\right)$ eram trabalhadores prévios e $8,3 \%\left(\mathrm{IC}_{95 \%} 6,0 ; 12,0\right)$ eram não trabalhadores. Entre os entrevistados, 339 $(89,9 \%)$ participantes tinham idade inferior a 16 anos, e destes, $82,3 \%$ eram trabalhadores atuais (dados não apresentados em tabelas). Houve pouca diferença entre os percentis de trabalhos conforme idade e sexo. Sobre raça/cor da pele, as diferenças não demonstraram significância estatística: dos escolares de raça/cor da pele branca, $10,2 \%$ não trabalhavam, $82,5 \%$ trabalhavam e $7,3 \%$ tiveram trabalho prévio; e dos não brancos, $8,8 \%$ não trabalhavam, $81,8 \%$ trabalhavam e $9,4 \%$ tiveram trabalho prévio. Entre os escolares que avaliaram sua saúde como excelente, 20,3\% não trabalhavam, 70,3\% trabalhavam e $9,5 \%$ tiveram trabalho prévio, enquanto dos que referiram sua saúde como regular ou ruim, $4,9 \%$ não trabalhavam, $82,7 \%$ trabalhavam e 12,4\% tiveram trabalho prévio $(\mathrm{p}<0,001)$ (Tabela 1$)$.

A Tabela 2 descreve características de trabalho dos jovens classificados como trabalhadores atuais. Quase 
Tabela 1 - Descrição do perfil de trabalho dos adolescentes da zona rural ( $n=377)$ segundo variáveis sociais, demográficas e de saúde, Barão do Triunfo, Rio Grande do Sul, 2010

\begin{tabular}{|c|c|c|c|c|}
\hline \multirow{3}{*}{ Variáveis } & \multicolumn{3}{|c|}{ Trabalho } & \multirow{3}{*}{$p$-valor ${ }^{a}$} \\
\hline & \multicolumn{2}{|c|}{ Atual } & \multirow{2}{*}{$\begin{array}{c}\text { Prévio } \\
\underset{\text { Sim }}{\%} \\
\end{array}$} & \\
\hline & $\begin{array}{c}\text { Não } \\
\%\end{array}$ & $\underset{\%}{\operatorname{Sim}}$ & & \\
\hline Perfil de trabalho & 8,3 & 82,1 & 9,6 & \\
\hline Idade (em anos) & & & & 0,740 \\
\hline $10-13$ & 10,0 & 80,8 & 9,2 & \\
\hline $14-15$ & 9,1 & 85,4 & 5,5 & \\
\hline$\geq 16$ & 8,3 & 80,6 & 11,1 & \\
\hline Sexo & & & & 0,631 \\
\hline Masculino & 9,6 & 80,8 & 9,6 & \\
\hline Feminino & 9,7 & 83,5 & 6,8 & \\
\hline Raça/cor da pele & & & & 0,707 \\
\hline Branca & 10,2 & 82,5 & 7,3 & \\
\hline Não branca & 8,8 & 81,8 & 9,4 & \\
\hline Autoavaliação de saúde ${ }^{b}$ & & & & $<0,001$ \\
\hline Excelente & 20,3 & 70,3 & 9,5 & \\
\hline Muito boa & 7,9 & 88,1 & 4,0 & \\
\hline Boa & 5,4 & 90,1 & 4,5 & \\
\hline Regular/ruim & 4,9 & 82,7 & 12,4 & \\
\hline
\end{tabular}

a) Teste do qui quadrado para heterogeneidade.

b) $n=342$.

metade deles $(45,4 \%)$ começou a trabalhar entre os 11 e os 12 anos de idade, e a maioria trabalhava em casa $(87,2 \%)$. Entre os tipos de atividade realizados, os mais frequentes foram os serviços domésticos/cuidado de irmãos (41,6\%) e os de agricultura/pecuária (42,1\%). Em relação à carga de trabalho, $23,6 \%$ relataram trabalhar nove ou mais horas diárias e $56,5 \%$ durante seis ou sete dias por semana. Aproximadamente $68,0 \%$ dos jovens relataram receber alguma remuneração em dinheiro por seu trabalho.

Em relação aos comportamentos de risco à saúde, 0 percentual de consumo de tabaco foi semelhante entre os três grupos de perfil de trabalho, diferentemente do consumo de álcool, referido por $29,5 \%$ dos trabalhadores atuais, $12,9 \%$ dos trabalhadores prévios e $17,7 \%$ dos não trabalhadores $(\mathrm{p}=0,06)$. As prevalências de excesso de peso e inatividade física no lazer não diferiram significativamente entre si, conforme o perfil de trabalho (Tabela 3).

Tendo em vista o baixo número de jovens nas categorias de trabalho prévio $(\mathrm{n}=31)$ e não trabalhadores $(\mathrm{n}=36)$, analisou-se a prevalência dos fatores de risco comparando-se trabalhadores atuais com todos os não trabalhadores no momento do estudo (trabalhadores prévios e não trabalhadores). A Figura 1 revela que 0 consumo de bebida alcoólica foi maior entre os trabalhadores atuais (29,5\%), quando comparados aos não trabalhadores $(15,4 \%)(\mathrm{p}=0,01)$. Essa associação se manteve mesmo após ajuste para sexo e idade dos escolares, e para renda familiar e escolaridade dos pais (dados não apresentados em tabelas). A ausência de associação entre excesso de peso, inatividade física no lazer e consumo de tabaco nos últimos 30 dias, e o trabalho atual, observada nas análises anteriores, manteve-se também nesta análise.

\section{Discussão}

A prevalência de trabalho entre a população estudada foi elevada: oito em cada dez escolares (10-16 anos) referiram atividade laboral, e a maioria das tarefas executadas por eles se caracterizavam como trabalho infanto-juvenil. A autoavaliação de saúde referida pelos adolescentes como excelente ou regular/ruim diferiu entre os perfis de trabalho. No que concerne aos comportamentos de risco, o consumo de bebidas alcoólicas 
Tabela 2 - Perfil de adolescentes trabalhadores, moradores da zona rural $(n=310)$, segundo variáveis relacionadas às características da atividade laboral, Barão do Triunfo, Rio Grande do Sul, 2010

\begin{tabular}{lll}
\hline Variáveis independentes & $\mathbf{n}$ & $\%$ \\
\hline Idade (em anos) em que começou a trabalhar & & 38,5 \\
\hline$<11$ & 145 & 45,4 \\
$11-12$ & 171 & 16,2 \\
$\geq 13$ & 61 & 87,2 \\
\hline Local da atividade laboral & 286 & 9,8 \\
\hline Casa & 32 & 3,0 \\
Fora de casa & 10 & 41,6 \\
Ambos & & 42,1 \\
\hline Tipo de atividade laboral & 140 & 16,3 \\
\hline Serviços domésticos/cuidado de irmãos & 142 & \\
Serviços de agricultura/pecuária & 55 & 43,8 \\
Outras atividades & & 32,6 \\
\hline Número de horas trabalhadas por dia & 165 & 23,6 \\
\hline$\leq 4$ & 123 & 31,6 \\
$5-8$ & 89 & 68,4 \\
\hline$\geq 9$ & & 43,5 \\
\hline Número de dias de trabalho por semana & 164 & 56,5 \\
\hline $1-5$ & 213 & \\
\hline $6-7$ & & 229 \\
\hline Remuneração em dinheiro & 106 & \\
\hline Sim & & \\
\hline
\end{tabular}

foi associado ao trabalho. Ressalta-se que o trabalho infanto-juvenil expõe a riscos e perigos atuais e futuros, não levados em conta pela família e gestores.

Entre os adolescentes da Pesquisa Nacional de Saúde do Escolar (PeNSE) realizada em 2015, precisamente entre aqueles que autorreferiram seu estado de saúde como ruim, a maioria consistiu de trabalhadores, na análise bruta, comparados aos não trabalhadores, um achado que perdeu poder na análise ajustada, ${ }^{20}$ similarmente aos achados desta pesquisa. Com base em revisão de literatura recente, 0 trabalho infanto-juvenil continua associado - negativamente - à saúde física e psicológica das crianças envolvidas. ${ }^{21}$ Estudos também referem que a zona rural apresenta maior percentual de trabalho infanto-juvenil e resultados mais impactantes à saúde dos indivíduos. ${ }^{1,5,8} 0$ consumo de bebida alcoólica no último mês mostrou-se maior entre os escolares trabalhadores atuais, comparados a seus pares não trabalhadores, no município. Cabe ressaltar que 0 uso de álcool na adolescência pode ter diversos impactos negativos na saúde: depressão, comportamento suicida, acidentes de automóvel, dependência de álcool na vida adulta, entre outros. ${ }^{22}$

Alguns fatores socioculturais podem explicar, ainda que parcialmente, a associação encontrada. A possibilidade de os escolares trabalhadores se sentirem mais autônomos e mais próximos do mundo adulto, por exemplo, pode refletir um padrão cultural local em que o consumo de bebida com álcool é valorizado. ${ }^{3} \mathrm{Em}$ Barão do Triunfo, o consumo de bebida alcoólica é constantemente incentivado em momentos de integração e lazer (como festas religiosas e bailes noturnos) entre crianças, adolescentes e adultos, a despeito de haver restrições para a venda dessas bebidas a menores de idade - cumpre observar, sem a devida fiscalização. Embora o presente estudo não pretendesse avaliar os locais de consumo, a grande participação das crianças e adolescentes nesses eventos e 0 incentivo constante pode ser um fator desencadeador para o resultado encontrado, especialmente entre os trabalhadores, muitos dos 
Tabela 3 - Comportamentos de risco à saúde conforme perfil de trabalho dos adolescentes da zona rural ( $n=377)$, por análise bruta e ajustada, Barão do Triunfo, Rio Grande do Sul, 2010

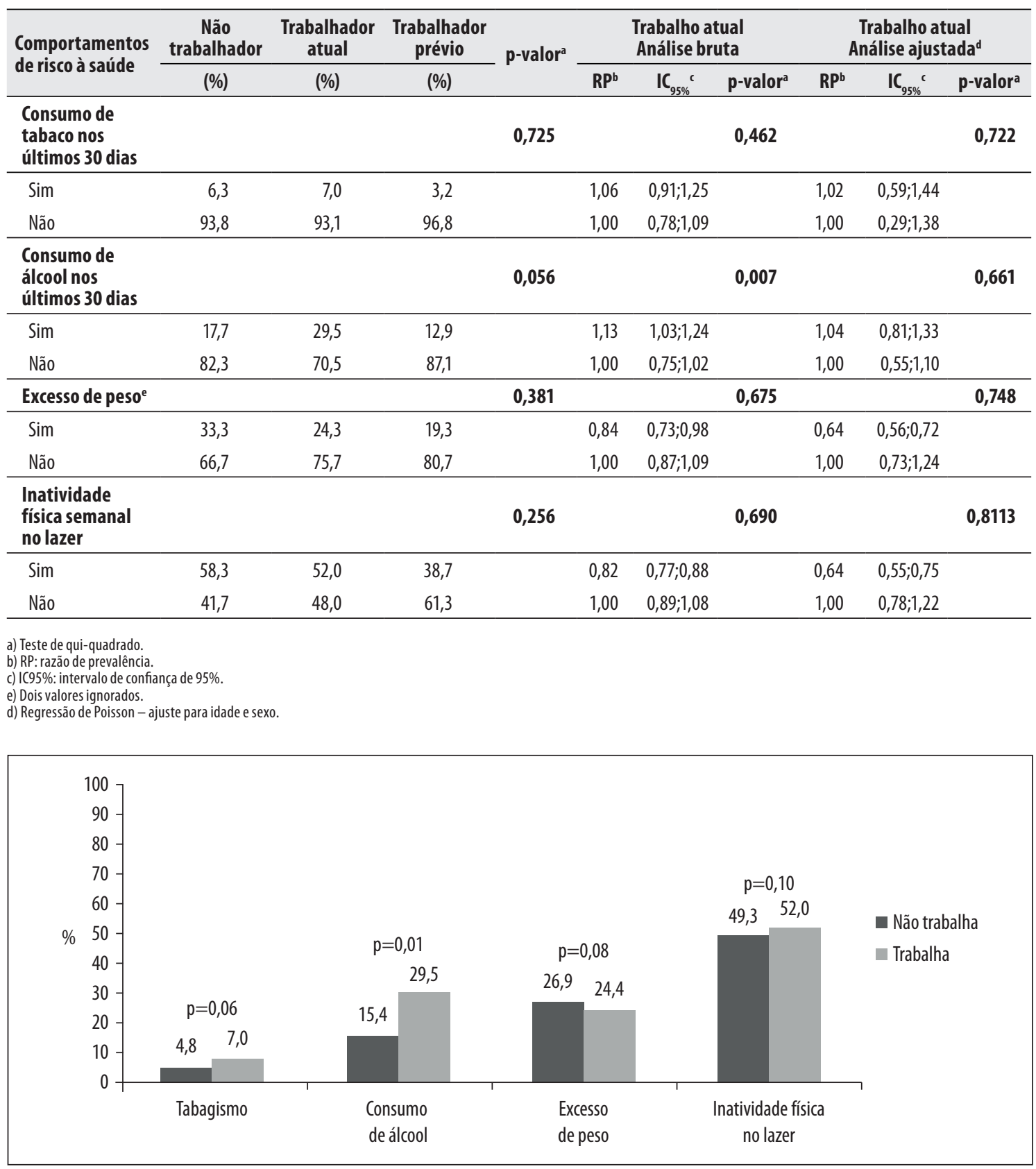

a) Regressão de Poisson.

Figura 1 - Prevalência de comportamentos de risco entre os adolescentes da zona rural, trabalhadores atuais e não trabalhadores, ajustada por sexo e idade, Barão do Triunfo, Rio Grande do Sul, 2010 
quais em condições de arcar com esse tipo de despesa. Motivações como curiosidade, busca por prazer, animação, redução da timidez, ansiedade (decorrente das condições de trabalho ou do compromisso precoce com a função laboral), aceitação dos amigos e padrões de socialização associados ao trabalho e ao mundo adulto ${ }^{22}$ podem estimular o uso de álcool. Junta-se a isso a influência cultural histórica da imigração: a população local é de origem predominantemente italiana e alemã, cujas identidades étnico-culturais se associam, respectivamente, ao hábito do consumo de vinho e cerveja.

0 comportamento encontrando corrobora os resultados do I Levantamento Nacional sobre os Padrões de Consumo de Álcool na População Brasileira, no que se refere à comparação entre os adolescentes (14-19 anos) das zonas urbanas e os de localidades rurais brasileiras. Madruga et al. ${ }^{23}$ verificaram que a prevalência de abuso ou dependência do álcool era semelhante entre adolescentes de ambas as áreas. 0 principal fator determinante desse comportamento não é o local de moradia, e sim o contexto mais amplo em que os escolares estão inseridos.

Ressalta-se que, na zona urbana, a ingestão de álcool é incentivada pela presença, em vários espaços, da publicidade, ${ }^{24}$ além desta, na zona rural, outros componentes e atores influenciam no consumo da substância, com resultados semelhantes. Todavia, as consequências desse comportamento ao longo da vida dos moradores de localidades rurais podem ser mais negativas, dado o menor acesso a uma série de recursos em saúde, apoio social e educação, comparados aos mesmos recursos disponíveis na zona urbana. Em países desenvolvidos, os jovens rurais apresentam episódios de embriaguez e consumo de risco mais frequentes que os da zona urbana. ${ }^{25}$

Contrariamente ao esperado, o consumo de tabaco não se mostrou associado ao perfil de trabalho. Apesar de os trabalhadores referirem consumo de tabaco maior que os não trabalhadores, não foi confirmada diferença estatistica. Entretanto, as prevalências foram similares às encontradas entre os investigados na PeNSE/2015 $(5,6 \%) .{ }^{26}$ Outro estudo, este com uma coorte de adolescentes, demonstrou a forte relação entre trabalhar e uso de tabaco. ${ }^{27}$ Em Barão do Triunfo, muitos dos agricultores são plantadores de tabaco, pelo que as crianças e adolescentes trabalhadores na agricultura lidam diretamente com a substância ou conhecem muito sobre esse tipo de cultivo. 0 contato frequente pode encorajar ao uso, a despeito da existência de medidas regulatórias para a publicidade e venda de tabaco. ${ }^{13}$
0 excesso de peso e a atividade física no lazer não se mostraram relacionados ao perfil de trabalho. Estudo de revisão de literatura não encontrou relação do trabalho com o estado nutricional entre jovens. Entretanto, estudos com trabalhadores de setores não sedentários (por exemplo, o setor agrícola ou o da construção) apontaram associação entre 0 trabalho infanto-juvenil e menor altura e peso, com a mesma direção e magnitude..$^{28}$ A prevalência de inatividade física no lazer entre jovens brasileiros é geralmente alta, ${ }^{26}$ justificada pelas barreiras existentes e relatadas pelos próprios adolescentes, como falta de companhia, tempo, dinheiro e/ ou preferência por outras atividades. ${ }^{27} \mathrm{Na}$ zona rural, a falta de espaços públicos específicos para a prática de atividade física pode ter influenciado 0 achado deste estudo, similar aos encontrados para escolares de outras regiões do país. ${ }^{29}$

Cabe mencionar que, na fase da vida avaliada nesta pesquisa, a carga de trabalho pode ter modificado fatores endócrinos, relacionados ao ganho de peso, e as jornadas laborais, por sua vez, previnam - devido ao esforço físico - 0 acúmulo de gordura abdominal. Todavia, a atividade laboral que exige esforço físico, em geral, leva ao maior gasto calórico e ao aumento de peso com 0 passar dos anos. ${ }^{30}$

Algumas limitações inerentes ao estudo, como o delineamento transversal, impedem a avaliação de temporalidade nas associações encontradas. Ademais, não foram investigados o tempo de início dos comportamentos avaliados, tampouco se o consumo de álcool e tabaco era de alto risco à saúde. Apesar de as perdas e recusas de escolares somarem menos de $10 \%$, é possível que os adolescentes ausentes da escola estivessem trabalhando, o que caracterizaria viés de seleção. Outros aspectos do trabalho infanto-juvenil não foram capturados por esta pesquisa, particularmente o das crianças e adolescentes envolvidos em atividades ilícitas, como prostituição e tráfico de drogas. Bastante mais perigosas, essas atividades, possivelmente, seriam determinantes para que essas crianças e jovens não se encontrassem na escola.

Outrossim, o tamanho amostral do estudo é pequeno e pouco heterogêneo, embora represente a totalidade dos escolares do município. Isso impediu, nas análises, a estratificação dos comportamentos de risco à saúde por sexo. Não obstante, destaca-se que o estudo é um dos poucos sobre o tema, não se tendo encontrado pesquisas brasileiras que relacionassem ao trabalho de crianças e adolescentes do meio rural todos os comportamentos de 
risco à saúde observados aqui. Alguns estudos consultados relacionam um, dois ou três desses comportamentos com a atividade laboral do adolescente, a maior parte deles focados em populações do meio urbano e não rural.

Conclui-se que foi elevada a prevalência de adolescentes escolares trabalhadores em Barão do Triunfo, em sua maioria em ocupações caracterizadas como trabalho infanto-juvenil. Houve predomínio dos serviços domésticos e da agricultura, e o comportamento de risco à saúde mais prevalente entre os trabalhadores atuais foi o consumo de bebida alcoólica nos últimos 30 dias. Estes achados reforçam a necessidade de ampliar a abrangência das políticas públicas destinadas à erradicação do trabalho infanto-juvenil e de promoção da saúde de jovens escolares, especialmente de moradores de zona rural. Ações conjuntas desenvolvidas com a família, governo, socieda-

\section{Referências}

1. International Labour Organization (ILO). Global estimates of child labour: Results and trends, 20122016 Internet . 2017. Available from: https://www.ilo. org/wcmsp5/groups/public/---dgreports/---dcomm/ documents/publication/wcms_575499.pdf\%0Ahttps:// www.ilo.org/global/publications/books/WCMS_575499/ lang--en/index.htm

2. Facchini LA. Trabalho infantil em Pelotas : perfil ocupacional e contribuição à economia Child labor in Pelotas : occupational characteristics and contribution to the economy. Ciências de Saúde Coletiva Internet 20003;8(4):953-61. Available from: http://www.scielo. br/pdf/csc/v8n4/a17v8n4.pdf

3. Radfar A, Asgharzadeh SAA, Quesada F, Filip I. Challenges and perspectives of child labor. Ind Psychiatry J 2018;27(1):17-20.

4. Marin JOB. 0 Agronegócio e o problema do trabalho infantil. Rev Sociol e Polit 2010;18(35):189-206.

5. Fassa AG, Facchini LA, Dall'agnol MM, Christiani DC. Child labor and health: Problems and perspectives. Int $J$ Occup Environ Health 2000;6(1):55-62.

6. Presidência da República B. Decreto n. 6.481, 12 de junho de 2008. Regulamenta os artigos $3^{\circ}$, alínea "d", e $4^{\circ}$ da Convenção 182 da Organização Internacional do Trabalho (OIT) que trata da proibição das piores formas de trabalho infantil e ação imediata para sua eliminação, aprovada .2008.

7. Presidência da República B. Lei no 8.069; de 13 de julho de 1990. Dispõe sobre o Estatuto da Criança e do Adolescente e dá outras providências. 2019. de civil e terceiro setor são fundamentais para erradicar o trabalho infantil e melhorar as condições de trabalho e de saúde dos jovens, pautadas em políticas mais amplas ou adaptadas às questões sociais, ambientais e culturais de cada localidade ou população específica.

\section{Contribuição dos autores}

Raphaelli CO e Azevedo MR contribuíram na concepção e delineamento do artigo, análise e interpretação dos dados e redação da primeira versão do manuscrito. Gonçalves He Hallal PC contribuíram na análise e interpretação dos dados e revisão crítica do manuscrito. Todos os autores aprovaram a versão final e são responsáveis por todos os aspectos do trabalho, incluindo a garantia de sua precisão e integridade.

8. Brasil. Pesquisa Nacional por Amostra de Domicílios : síntese de indicadores 2014/ IBGE, Coordenação de Trabalho e Rendimento Internet . 2015. Available from: http://biblioteca.ibge.gov.br/visualizacao/livros/ liv94935.pdf

9. Wright S, Marlenga B, Lee BC. Childhood agricultural injuries: An update for clinicians. Curr Probl Pediatr Adolesc Health Care Internet 2013;43(2):20-44. Available from: http://dx.doi.org/10.1016/j. cppeds.2012.08.002

10. Rauscher K, Runyan C. Adolescent occupational fatalities in north carolina (1990-2008): An investigation of child labor and osha violations and enforcement. New Solut 2013;22(4):473-88.

11. Sousa OMCG de, Alberto M de FP. Early work and children's and adolescents' schooling process. Psicol em Estud 2008;13(4):713-22.

12. Souza DPO de, Areco KN, Silveira Filho DX da. Álcool e alcoolismo entre adolescentes da rede estadual de ensino de Cuiabá, Mato Grosso TT - Alcohol and alcoholism among Brazilian adolescent public-school students. Rev Saude Publica Internet 2005;39(4):585-92.

13. Maldonado RM, Pedrão LJ, Castillo MMA, García KSL, Rodríguez NNO. Auto-estima, auto-eficácia percebida, consumo de tabaco e álcool entre estudantes do ensino fundamental, das áreas urbana e rural, de monterrey, Nuevo León, México. Rev Lat Am Enfermagem 2008;16(spe):614-20.

14. De Rezende LFM, Azeredo CM, Canella DS, Claro RM, De Castro IRR, Levy RB, et al. Sociodemographic 
and behavioral factors associated with physical activity in Brazilian adolescents. BMC Public Health 2014;14(1):1-11.

15. Shoman AE, Mostafa NS, Musslem AA. Nutritional status and parasitic infestation among working children in a village in Egypt: A comparative study. J Egypt Public Health Assoc 2015;90(2):80-4.

16. Instituto Nacional de Pesquisas e Estudos Anísio Teixeira I. Censo Escolar 2010. 2010;2010:1-42.

17. Carlini EL de A, Noto AR, Carlini CM de A, Locatelli DP, Abeid LR, Amato T de C, et al. VI Levantamento Nacional sobre o Consumo de Drogas Psicotrópicas entre Estudantes do Ensino Fundamental e Médio das Redes Publica e Privada de Ensino nas 27 Capitais Brasileiras. 2010.

18. Must A, Dallal GE, Dietz WH. Reference data for obesity: 85th and 95th percentiles of body mass index (wt/ ht2) and triceps skinfold thickness. Am J Clin Nutr 1991;53(4):839-46.

19. Bastos JP, Araújo CLP, Hallal PC. Prevalence of insufficient physical activity and associated factors in Brazilian adolescents. J Phys Act Heal 2008;5(6):777-94.

20. Malta DC, De Oliveira MM, Machado IE, Prado RR, Stopa SR, Crespo CD, et al. Characteristics associated to a poor selfrated health in Brazilian adolescents, National Adolescent School-based Health Survey, 2015. Rev Bras Epidemiol 2018;21(Suppl 1).

21. Ibrahim A, Abdalla SM, Jafer M, Abdelgadir J, De Vries N. Child labor and health: A systematic literature review of the impacts of child labor on child's health in lowand middle-income countries. J Public Heal (United Kingdom) 2019;41(1):18-26.

22. Leeman RF, Hoff RA, Krishnan-Sarin S, PatockPeckham JA, Potenza MN. Impulsivity, Sensation-
Seeking and Part-Time Job Status in Relation to Substance Use and Gambling in Adolescents. J Adolesc Heal 2014:54(4):460-6.

23. Madruga CS, Laranjeira R, Caetano R, Pinsky I, Zaleski $\mathrm{M}$, Ferri CP. Use of licit and illicit substances among adolescents in Brazil - A national survey. Addict Behav Internet 2012;37(10):1171-5. Available from: http:// dx.doi.org/10.1016/j.addbeh.2012.05.008

24. Faria R, Vendrame A, Silva R, Pinsky I. Association between alcohol advertising and beer drinking among adolescents. Rev Saude Publica 2011;45(3):441-7.

25. Legleye S, Janssen E, Spilka S, Le Nézet 0, Chau N, Beck F. Opposite social gradient for alcohol use and misuse among French adolescents. Int J Drug Policy 2013;24(4):359-66.

26. Brasil. Ministério da Saúde. Pesquisa nacional de saúde do escolar : 2015. Internet .2016. Available from: https://biblioteca.ibge.gov.br/visualizacao/livros/ liv97870.pdf

27. Dias DF, Loch MR, Ronque ERV. Perceived barriers to leisure-time physical activity and associated factors in adolescents. Cienc e Saude Coletiva 2015;20(11):3339-50

28. Aransiola TJ, Justus M. Child labor hazard on mental health: Evidence from Brazil. J Ment Health Policy Econ 2018;21(2):49-58.

29. Figueiredo VC, Szklo AS, Costa LC, Kuschnir MCC, Da Silva TLN, Bloch KV, et al. ERICA: Smoking prevalence in Brazilian adolescents. Rev Saude Publica 2016;50(supl 1):1s-10s.

30. Yamada Y, Kameda M, Noborisaka Y, Suzuki H, Honda M, Yamada S. Excessive fatigue and weight gain among cleanroom workers after changing from an 8-hour to a 12-hour shift. Scand J Work Environ Heal 2001;27(5):318-26. 


\section{Abstract}

objective: to evaluate association between work and bealth risk behaviors among rural schoolchildren. Methods: this was a cross-sectional study with 5th to 8th grade elementary and middle school students attending municipal schools in Barão do Triunfo, RS, Brazil in 2010; a self-administered questionnaire was used; their work profile was characterized as 'current workers', 'former workers', and 'non-workers'; health risk behaviors examined were smoking, alcohol consumption, overweight, and physical inactivity ( $\leq 300$ minutes per week). Results: of the 339 participants under 16 years old (89.9\%), 82.3\% were current workers; their predominant work was domestic services (39.8\%), and farming (29.9\%); $24.8 \%$ of students were overweight, $51.2 \%$ were inactive during leisure, $6.6 \%$ smoked, and $27.0 \%$ had consumed alcobol in the last 30 days; alcobol consumption was higher among workers than non-workers ( $p=0.02)$. Conclusion: eight out of ten schoolchildren worked, most of them as child labor; the most frequent health risk behavior was alcohol consumption.

Keywords: Adolescent Behavior; Health; Child Labor; Alcoholic Beverages; Cross-Sectional Studies.

\section{Resumen}

Objetivo: evaluar la asociación entre el trabajo y los comportamientos de riesgo a la salud entre los escolares de zona rural. Métodos: estudio transversal con estudiantes de quinto a octavo grado deprimaria en las escuelas municipales de Barão do Triunfo, RS, Brasil, en 2010; en cuestionario autoaplicable, se caracterizó el perfil de trabajo como 'trabajadores actuales', 'trabajadoresprevios' y 'no trabajadores'; los comportamientos de riesgo a la salud fueron el tabaquismo (sí; no) y el consumo de alcobol (si; no) en el último mes, exceso depeso e inactividad física. Resultados: entre los 339 participantes menores de 16 años (89,9\%), el 82,3\% eran trabajadores; entre los trabajadores, predominaron los servicios domésticos $(39,8 \%)$ y la agricultura (29,9\%); 24,8\% de los estudiantes tenía sobrepeso, 51,2\% estaba inactivo en el tiempo libre, 6,6\% fumabay 27,0\% consumió alcobol en los últimos 30 días; este consumo fue mayor entre los trabajadores que entre los no trabajadores $(p=0,02)$. Conclusión: ocho de cada diez escolares trabajaba, y la mayoría fue de niños y adolescentes; el comportamiento de mayor riesgo entre ellos fue el alcobol.

Palabras clave: Conducta del Adolescente; Salud; Trabajo Infantil; Bebidas Alcohólicas; Estudios Transversales.

Recebido em 20/08/2019

Aprovado em 23/03/2020 